The Author(s), 2020. Published by Cambridge University Press on behalf of the University of Arizona. This is an Open Access article, distributed under the terms of the Creative Commons Attribution licence (http://creativecommons.org/licenses/by/4.0/), which permits unrestricted re-use, distribution, and reproduction in any medium, provided the original work is properly cited.

\title{
VOLUMES OF WORTH_DELIMITING THE SAMPLE SIZE FOR RADIOCARBON DATING OF PARCHMENT
}

\author{
Tuuli M Kasso $^{1 * \text { (D) }} \cdot$ Markku J Oinonen $^{2}$ (D) $・$ Kenichiro Mizohata ${ }^{2,3} \cdot$ Jaakko K Tahkokallio $^{4}$ \\ Tuomas M Heikkilä ${ }^{5}$ \\ ${ }^{1}$ The Globe Institute, University of Copenhagen, Øster Farimagsgade 5, 1350 Copenhagen, Denmark \\ ${ }^{2}$ Laboratory of Chronology, Finnish Museum of Natural History (LUOMUS), 00014 University of Helsinki, Finland \\ ${ }^{3}$ Accelerator Laboratory, Faculty of Sciences, University of Helsinki, 00014 University of Helsinki, Finland \\ ${ }^{4}$ National Library of Finland, 00014 University of Helsinki, Finland \\ ${ }^{5}$ Faculty of Theology, University of Helsinki, 00014 University of Helsinki, Finland
}

\begin{abstract}
Medieval manuscripts are invaluable archives of the written history of our past. Manuscripts can be dated and localized paleographically, but this method has its limitations. The Fragmenta membranea manuscript collection at the National Library of Finland has proved difficult to date using paleographic methods. Radiocarbon dating has been applied to manuscripts of parchment before, but a systematic protocol for radiocarbon dating of parchment has not been established with a minimally destructive sampling strategy. In this work, we have established a radiocarbon dating procedure for parchments combining a clean-room based chemical pretreatment process, elemental analyzer combustion, automatic graphitization and accelerator mass spectrometry (AMS) measurements to reduce the AMS target size from a typical $1 \mathrm{mg}$ of carbon. Prolonged acid treatment resulted in improved dating accuracy, since this is consistent with the manufacturing process of medieval parchment involving a lime bath. Two different combustion processes were compared. The traditional closed tube combustion (CTC) method provided a well-established though labor-intensive way to produce $1 \mathrm{mg}$ AMS targets. The Elemental Analyzer-based process (EA-HASE, Elemental Analyzer Helsinki Adaptive Sample prEparation line), is designed for fast combustion and smaller sample sizes. The EA-HASE process was capable of reproducing the simulated radiocarbon ages of known-age samples with AMS graphite target sizes of $0.3 \mathrm{mg}$ of carbon, corresponding to a $3 \mathrm{~mm}^{2}$ area of a typical medieval parchment. The full potential of the process to go down to as little as $50 \mu \mathrm{g}$ will be further explored in the future in parallel to studies of sample-specific contamination issues.
\end{abstract}

KEYWORDS: contamination, graphitization, parchment, radiocarbon AMS dating, sample size.

\section{INTRODUCTION}

Radiocarbon $\left({ }^{14} \mathrm{C}\right)$ dating of archaeological objects and organic remains obtained from an archaeological site is the most commonly dating method in archaeology, however this still causes concern due to its destructive approach. To resolve this, further development is needed to improve the method of ${ }^{14} \mathrm{C}$ dating by delimiting the sample size, joined to the removal of contamination, which can prohibit accurate dating. A typical target size of carbon needed for ${ }^{14} \mathrm{C}$ dating with accelerator mass spectrometry (AMS) is $1 \mathrm{mg}$. However, this is not the size of the whole sample itself-since the sample is not $100 \%$ carbondepending on the material and its quality, usually more of the sample material is needed in order to ensure the necessary target size. The sample sizes of parchment mentioned in ${ }^{14} \mathrm{C}$ dating research vary, and are often not mentioned at all. For parchment, we estimated that such a $1 \mathrm{mg}$ AMS target size corresponds typically to $\sim 16 \mathrm{~mm}^{2}$ area of material, approximately $4 \times 4 \mathrm{~mm}$. Medieval manuscripts hold indispensable value, and any sampling should be minimally destructive, as with any cultural historical material. Therefore, ${ }^{14} \mathrm{C}$ dating results from sample sizes with less than $1 \mathrm{mg}$ of carbon should be pursued.

Made from the skin of animals, usually cattle-, goat-, or sheepskin, parchment was used in Europe for making books and documents from as early as the fifth century BC up to the

\footnotetext{
*Corresponding author. Email: tuuli@palaeome.org.
} 
early-modern era, used as the main writing medium for some 1000 years from fourth century AD onwards. Although manuscripts can be dated and localized paleographically with varying accuracy, ${ }^{14} \mathrm{C}$ dating can corroborate this information as an absolute dating method. This is the case with the Fragmenta membranea collection at the National Library of Finland, a manuscript fragment collection of little over 9300 leaves containing material from the early eleventh century up to the mid-sixteenth century. Since the collection items are fragments of books from a geographical region (Sweden and Finland) which is paleographically poorly charted, manuscript scholars have struggled to date much of this material. Typically, the fragments have been dated within a century, with dates of 150 years and even two centuries appear commonly in the collection catalogues. Thus, although ${ }^{14} \mathrm{C}$ dating precision also varies due to changes in the atmospheric ${ }^{14} \mathrm{C}$ content through ages, it should provide comparable natural scientific estimates for the fragments. The problem with ${ }^{14} \mathrm{C}$ dating such archival material lies of course in the destructive nature of the method, and minimizing the sample size is the key issue in applying it to this collection.

The challenge in ${ }^{14} \mathrm{C}$ dating any smaller sample size than $1 \mathrm{mg}$ of carbon is that the amount of contamination in the sample becomes more relevant. Therefore, the procedure must be monitored throughout and all carbon contamination, modern and old, has to be avoided to obtain as accurate results as possible. The key factor to removing contamination is the chemical pretreatment process, where the samples undergo before dating to remove all external or exogenous contaminating material, without destroying the original sample material excessively. The chemical procedure in this paper was designed based on the previous research on ${ }^{14} \mathrm{C}$ dating of parchment and was moved to a clean room environment. The clean room was included to exclude potential air-borne contaminants on the small samples deriving from dust particles during the handling of the samples. After the pretreatment process when the sample is combusted, a typical additional concern is the possibility of leaks during the sample $\mathrm{CO}_{2}$ processing, as it can expose the sample to modern atmospheric $\mathrm{CO}_{2}$.

However, one of the most significant potential contamination in parchment is derived from its manufacturing process. Animal skins are treated with lime as a part of the dehairing process, and the parchment smoothed with pumice when dry. Lime introduces calcium carbonate, $\mathrm{CaCO}_{3}$, a fossil contamination to the material. To remove this, an acid-based solvent must be applied. Parchment was occasionally reused as palimpsest and in such cases the revitalizing of the medium may have been done with a wash of milk, wine, or other products, but the residual effect of this process is marginal compared to the $\mathrm{CaCO}_{3}$ or heavy conservation with modern materials. Substances derived from conservation treatments are frequent contaminants in ${ }^{14} \mathrm{C}$ samples, and often are imperceptible to the naked eye. Some adhesives used in conservation of parchment are gelatin and parchment (mammalian animal) glue. The difficult to remove castor oil, which has been used to improve the visibility of faded ink, and modern synthetic polymers and acrylic resin solutions, such as versions of Paraloid ${ }^{\circledR}$, should always be noted for their possible presence in a sample due to their wide appliance in conservation.

In this work, we established a ${ }^{14} \mathrm{C}$ dating procedure for parchment combining clean-room based chemical pretreatment process, elemental analyzer combustion, automatized graphitization and AMS measurement to reduce the AMS target size from a typical $1 \mathrm{mg}$ of carbon. We used modern parchment samples for process development and demonstrated the capability of the method by using known-age medieval parchment manuscripts. 


\section{STATE OF THE ART IN PARCHMENT STUDIES}

In order to establish a method, which both eliminates modern and ancient carbon contamination and pursues a smaller sample size, it is crucial to study material with a known age. This allows the results to be analyzed and their quality evaluated. Modern parchment is one option for such a study, since it can be easily acquired and information about its precise manufacturing date is available. Modern material also has certain advantages for the development of the method. Because of the difference in the atmospheric ${ }^{14} \mathrm{C}$ content between past and modern era, older material cannot be dated as accurately as younger material from after 1950. This is due to the atomic bomb effect, providing an accurate calibration for ${ }^{14} \mathrm{C}$ dates for samples from the last 6 decades. On the other hand, studying only modern material can give us a distorted view: the material can be in a far better condition that would be expected in handling historical parchments.

A typical process to obtain a ${ }^{14} \mathrm{C}$ date contains several phases: the chemical pretreatment, combustion of the sample, conversion of resulting carbon dioxide to pure carbon (graphitization) and the ${ }^{14} \mathrm{C}$ measurement itself. Therefore, ${ }^{14} \mathrm{C}$ dating results can suffer from various errors and effects deriving from these several sources. The age of the material, errors in the measurements, faults from laboratory procedures, contamination and concentration of ${ }^{14} \mathrm{C}$ are the main causes of error. Also, the error entailed by the unknown age of the material must be considered, as some organic materials have possibly been dead or stored for a considerable time before being used by man in the past. Or, as in the case of manuscripts, reusing the material after decades or even centuries of its original creation-a palimpsest.

The major advantage of AMS is the detection efficiency, which has successfully resulted in moving towards smaller sample sizes, decreasing from grams to milligrams and now to micrograms. However, the smaller the sample, the more influence any remaining contamination will have on the ${ }^{14} \mathrm{C}$ dating. Fiona Brock (2013b) remarks (after Gupta and Polach 1985) how the amount and age of modern carbon (we assume $\mathrm{F}^{14} \mathrm{C}=1 / \mathrm{pMC}=1$ ) present in a sample affects the values of a 900-year-old BP sample: $1 \%$ of modern carbon contamination would affect the dating to $\sim 890$ years BP, while only $5 \%$ of the same contaminant would give a dating of $\sim 850$ years. If the contaminant is old carbon (we assume $\left.\mathrm{F}^{14} \mathrm{C}=0 / \mathrm{pMC}=0\right), 1 \%$ would result in an apparent age of $\sim 980$ years $\mathrm{BP}$ and $5 \%$ to $\sim 1320 \mathrm{BP}$. Errors in the laboratory procedures can be detected for example when doing inter-laboratory measurements from the same sample, and doing these tests should be increased. Currently the challenges are the extent of the dating time frame, as well as the problem of contamination.

There are various sources of contamination to a parchment sample that can derive from multiple sources. This can be direct, such as storage or conservation treatments often applied to cultural heritage objects, or indirect such as the limestone treatment deriving from the manufacturing process. Thus, chemical pretreatment for the samples is crucial. Subsequent sample combustion and graphitization processes may typically induce modern carbon contamination due to involvement of residual atmospheric carbon dioxide. Furthermore, the AMS measurement itself has additional statistical and systematic uncertainties. These all challenge the production of accurate and precise ${ }^{14} \mathrm{C}$ ages while working with minute samples.

Different pretreatment methods for parchment have been tried and compared in some previous studies. The standard acid-base-acid (ABA) or acid-alkaline-acid (AAA) treatment appears to be the most successful in eliminating contamination with minimum sample loss. The base wash 
or an additional bleach wash has been found effective, but following Brock (2013a) and Santos et al. (2010), any alkaline wash should be applied with caution as it can be very destructive, excluding especially fragile sample material. In the ${ }^{14} \mathrm{C}$ dating of fourteen Dead Sea scrolls by Bonani et al. (1992), parchment samples were treated with ABA. During the study, the impact of the regular treatment was evaluated, with several of the methods found to be destructive, as the material was lost completely for some samples. Notably, the sample loss is a substantial risk when handling parchment.

As there is a possibility that ABA is not sufficient to remove all contamination, for instance the widely applied Paraloid ${ }^{\circledR}$ B-72 used in conservation, which is soluble in acetone, applying a wash with this solvent prior to the ABA is recommended. Bleach may be effective for removing biological contamination but can result in serious loss of the sample material, and in any case the sample undergoes base as a part of the ABA. Hexane, acetone, combinations of methanol and chloroform have been tested, with acetone being the preferred solvent as it evaporates quickly from the sample. Donahue et al. (2002) further showed that a prior acetone wash is completely removed through a water rinse, with a $20-30 \%$ general loss of the sample overall during the pretreatment not depending on the acetone wash.

In contrast to Donahue et al. (2002), Brock (2013a) does not report on the loss of the total mass of the samples but notes only that the samples survived the chemical treatments better than expected. Considering that the treatments used by Brock prior to ABA are more comprehensive than those used by Donahue et al. (2012), some loss of the material can be assumed due to the washes. In the case of small samples, up to $30 \%$ loss of total material might still be acceptable, with less being ideal. When dating parchment with heavy lipid contamination, such as castor oil in the Dead Sea Scrolls, Rasmussen et al. (2009) report a low survival rate of the samples after the chemical pretreatment, which draws attention to challenges of highly contaminated samples.

Studies on ${ }^{14} \mathrm{C}$ dating of parchment rarely mention the size of the parchment samples, so the evaluation of the size of the samples was made based on the carbon content. As parchment is assumed to contain $\sim 90-95 \%$ collagen, of which approximately $35 \%$ is carbon, the C: $\mathrm{N}$ ratio (carbon-to-nitrogen ratio) can be used to represent the state of the collagen preservation in parchment samples in addition to contamination removal. The acceptable $\mathrm{C}: \mathrm{N}$ ratio for collagen in radiocarbon dating is $\sim 3.3$ (Brock 2013a, after van Klinken 1999).

As found by Brock et al. 2012, measuring the $\% \mathrm{~N}$ content of the sample material is the most effective way to select samples and avoid destroying samples that are unsuitable to ${ }^{14} \mathrm{C}$ dating. In the Laboratory of Chronology, $\% \mathrm{~N}$ and $\% \mathrm{C}$ values are measured with an elemental analyzer, which can measure both values together from the same sample. As the sample is completely destroyed during the measurement, this phase in evaluating whether the sample is suitable for ${ }^{14} \mathrm{C}$ dating or not, is problematic when considering the sample size. If the $\% \mathrm{~N}$ and $\% \mathrm{C}$ are not measured, there is a real possibility of destroying a sample completely prior to dating, thus destroying a sample in vain that could have provided important information other than just the age of the specimen. Hence, in dating small sample material, whether executing this phase or not prior to chemical pretreatments, must be carefully evaluated, and non-destructive methods for contamination removal (e.g. FTIR as Brock et al. 2018, and NIR) better explored.

During this study, the $\mathrm{C}: \mathrm{N}$ ratio was not measured before ${ }^{14} \mathrm{C}$ dating. The sampling of the medieval parchment was then made based on the theoretical assumed value of carbon in 
parchment: $90 \%$ collagen of which $35-37 \%$ is carbon, therefore $31.5 \%$ of the total mass. Later measuring the carbon-content and comparing it to a modern parchment, the values were very uniform between old and new material, ca. $42-44 \%$ in each. This indicates that the collagen quality of parchment preserved in library conditions may not significantly change over time, suggesting that the $\mathrm{C}: \mathrm{N}$ ratio is not a necessary step in sampling parchment.

\section{METHODS AND MATERIALS}

\section{Samples}

Three sample materials were used in this work. Modern parchment manufactured from the skin of a calf that died in October 2015 was used for initial tests of the chemical pretreatment process and for the first ${ }^{14} \mathrm{C}$-AMS analyses. Based on the assumption that the skin of the calf was stored the atmospheric ${ }^{14} \mathrm{C}$ contents for 12 months prior to its death, it was estimated based on Levin et al. (2013) that the ${ }^{14} \mathrm{C}$ content of parchment should have been $\sim 101.6$ pMC. The other samples used were two medieval documents with known original dates of writing of $\mathrm{AD}$ 1484 and AD 1506 to study our measurement process further.

\section{Clean Room for Sample Pretreatment}

Although the Laboratory of Chronology is using dedicated laboratory spaces and fume hoods for processing the ${ }^{14} \mathrm{C}$ samples, there is a minor risk that modern or fossil (i.e. ${ }^{14} \mathrm{C}$ dead) carbon contamination could be accidentally mixed with the samples through airborne particles, like dust, during sample preparation and particularly prior combustion. As the main aim of this study was to proceed towards small samples and AMS graphite masses significantly smaller than $1 \mathrm{mg}$, part of the work was to experiment with the chemical pretreatment procedure within a clean room for the first time.

A joint clean room infrastructure of Helsinki Institute of Physics and Department of Physics of the University of Helsinki is located close to the Laboratory of Chronology. This contains both class 1000 (or ISO 6/ISO 14644-1 and ISO 14698 standards) and 100 (or ISO 5) clean rooms. For this experiment, one fume hood from ISO 6 clean room was dedicated to the chemical pretreatment process for the samples. The ISO 6 class corresponds to a maximum of 35200 particles over $0.5 \mu \mathrm{m}$ in size per $\mathrm{m}^{3}$. This can be compared to typical room air that can have 1000 times more of such particles.

ISO 6 class operations involve using of dedicated hair cover, coverall and boots and careful wiping of all incoming material prior usage. Therefore, all the operations are slightly more time-consuming. Within this work, clean room pretreatments were very successful, without complications. After the chemical pretreatment process all the samples were allowed to dry overnight in a fume hood in the clean room. In the following morning the sample vials were closed and the samples were taken for combustion process.

\section{Chemical Pretreatment Process}

For the $\mathrm{A}$ to $\mathrm{C}$ series the applied chemical pretreatment was uniform:

1. Acetone wash in $45^{\circ} \mathrm{C}$ for $1 \mathrm{hr}$.

2. ABA: $\mathrm{HCl}(0.5 \mathrm{M}, 30 \mathrm{~min}) ; \mathrm{NaOH}(0.1 \mathrm{M}, 20 \mathrm{~min})$; $\mathrm{HCl}(0.5 \mathrm{M}, 1 \mathrm{hr})$. (Rinsing thoroughly with MilliQ ${ }^{\mathrm{TM}}$ water between and after each step.) 
The series were made with modern (A) and medieval $(\mathrm{B}, \mathrm{C})$ parchment with the combustion and graphitization with EA-HASE. Samples from modern parchment were run first in a separate AMS run giving relatively good results reproducing the modern ${ }^{14} \mathrm{C}$ content but suffering technical issues within AMS that lowered the statistical precision. The series $\mathrm{B}$ and $\mathrm{C}$ of medieval parchments provided results ranging between $276-931 \mathrm{BP}$, most of the results being too old. This was interpreted as resulting from $\mathrm{CaCO}_{3}$ contamination. Therefore, the acid treatment was changed to double the length of time for the remaining sample series $(\mathrm{D}, \mathrm{E})$.

For the final D and E series, the chemical pretreatment method was adjusted accordingly:

1. Acetone wash in $45^{\circ} \mathrm{C}$ for $1 \mathrm{hr}$.

2. ABA: $\mathrm{HCl}(0.5 \mathrm{M}, 60 \mathrm{~min})$; $\mathrm{NaOH}(0.1 \mathrm{M}, 30 \mathrm{~min}) ; \mathrm{HCl}(0.5 \mathrm{M}, 2 \mathrm{hr})$.

(Rinsing thoroughly with MilliQ ${ }^{\mathrm{TM}}$ water between and after each step.)

The duration of the $\mathrm{NaOH}$ bath was also lengthened by $10 \mathrm{~min}$, as the period of 20 min was found not harmful for the smallest sample. On the contrary, the base made the parchment samples swell, which helped the handling of the small samples. For the collagen to liquefy, the duration of $\mathrm{NaOH}$ treatment should be overnight, so consequently in theory there was no chance of losing a sample within $30 \mathrm{~min}$-nonetheless the samples were kept under close surveillance during the entire time.

\section{Sample Combustion and Graphitization}

Two different sample treatment methods were used for combusting and converting the combusted $\mathrm{CO}_{2}$ to graphite targets for ${ }^{14} \mathrm{C}$-AMS measurements. The main features of both the methods are provided below.

The traditional method to combust organic samples follows essentially the method B of ASTM D6866-18 standard (ASTM 2018). In short, pretreated sample is placed inside a pre-cleaned quartz tube sealed from the other end among $3 \mathrm{~g}$ of copper oxide grains. This tube is placed inside a larger pre-cleaned quartz tube which is evacuated to $<0.1$ mbar pressure and torch-sealed. This ampoule is placed in an oven for combustion in $900^{\circ} \mathrm{C}$ for at least $4 \mathrm{hr}$, generally overnight. The formed $\mathrm{CO}_{2}$ sample is cryogenically purified with liquid-N2 and cold (ca. $-85^{\circ} \mathrm{C}$ ) ethanol traps. The $\mathrm{CO}_{2}$ sample is subsequently converted to graphite with $\mathrm{Zn}$ and Fe catalysts (Slota et al. 1986) and pressed to an AMS target. This method is referred as Closed-Tube-Combustion (CTC) method.

The second method uses Elemental Analyzer (EA) combustion coupled with a LabVIEW controlled $\mathrm{CO}_{2}$ purification and graphitization line. The pretreated sample is placed in a tin cup (Elemental Microanalysis D1001) and combusted in EA (Thermo Scientific Flash $2000 \mathrm{NC})$. While the temperature of the combustion process $\left(1020^{\circ} \mathrm{C}\right)$ is sufficient enough to oxidize carbon to $\mathrm{CO}_{2}$, the used tin cups react with oxygen in an exothermic process rising the temperature briefly to $1800^{\circ} \mathrm{C}$. The formed $\mathrm{CO}_{2}$ is trapped cryogenically, purified and graphitized within the Helsinki Adaptive Sample PrEparation (HASE) line in the presence of zinc powder and iron catalyst (Palonen et al. 2014). This method is referred as EA-HASE.

Both graphitization lines of CTC and EA-HASE contain four graphitization reactors. As graphitization is a chemical reduction process and thus requires a certain amount 
( $>4 \mathrm{hr}$ ) of time, the essential gain is provided within EA-HASE by the combustion speed and purity. The full combustion process takes ca. $3 \mathrm{~min}$ instead of $>4 \mathrm{hr}$, reduces the time spent compared to $\mathrm{CTC}$ and limits also the potential modern $\mathrm{CO}_{2}$ contamination. Moreover, as the EA-HASE process has been optimized for repeatability, automatic control and small sample masses, we expect this to show up within the obtained results.

\section{${ }^{14} \mathrm{C}$ AMS Measurements}

The Laboratory of Chronology of University of Helsinki uses accelerator mass spectrometry (AMS) for ${ }^{14} \mathrm{C}$ measurements (Tikkanen et al. 2004). The Helsinki AMS can measure ${ }^{14} \mathrm{C}$ contents within $<0.2 \%$ precision after a major upgrade of the AMS control electronics in 2013. The AMS characteristics are controlled in Helsinki by using standard graphite samples, which provide ${ }^{14} \mathrm{C}$-free reference, to alarm any possible signals for needed maintenance. Corrections for isotopic fractionation are made by using the $\delta^{13} \mathrm{C}$ value measured by AMS. Therefore, the $\delta^{13} \mathrm{C}$ values contain potential isotopic fractionation due to graphitization and AMS measurement processes instead of being solely characteristic of samples and are not provided to avoid misinterpretations.

In this work, we aimed for moderate statistical precision $(1 \sigma)$ of $\pm 0.3-0.4$ percent modern carbon (pMC) yielding to a statistical precision $(1 \sigma)$ of $\pm 30-40{ }^{14} \mathrm{C}$ years for the ${ }^{14} \mathrm{C}$ age of the AD 1484 parchment sample. Both the D (CTC) and E (EA-HASE) measurement series were successful. The AMS machine backgrounds measured with natural graphite blanks pressed into AMS cathodes were $0.12 \mathrm{pMC}(54,300 \mathrm{BP})$ and $0.11 \mathrm{pMC}(54,400 \mathrm{BP})$ for CTC and EA-HASE series, respectively. The full process backgrounds (combustiongraphitization-AMS) were measured for the CTC process with natural graphite and for the EA-HASE process with fossil diesel samples. These showed slightly higher process background of $0.4 \mathrm{pMC}(44,300 \mathrm{BP})$ for CTC compared to $0.13 \mathrm{pMC}(53,100 \mathrm{BP})$ of EA-HASE. This indicates a slightly larger modern contamination resulting from the CTC process compared to the EA-HASE process. This is expected due to EA-HASE optimization for small samples. The difference observed within these individual measurements is coherent with the average long-term process background that is discussed separately (Uusitalo et al. 2020).

\section{Data Analysis}

We analyzed the measured ${ }^{14} \mathrm{C}$ contents by following the procedure used in the international radiocarbon intercomparison studies (Scott et al. 2010; $\mathrm{z}$ scores). The measured ${ }^{14} \mathrm{C}$ contents (pMC i.e. percent modern carbon) are compared to simulated $\mathrm{pMC}$ values $\left(\mathrm{pMC}_{\text {sim }}\right.$, mean, $\mathrm{N}=20$ ) corresponding to the assumed manufacturing year of parchment sample. The simulations were performed with R_Simulate command within OxCal software (Bronk Ramsey 2009). In particular, as R_Simulate produces a slightly different pMC every time, the simulations were performed 20 times for each measurement data. The differences were assessed through a two-sample $\mathrm{z}$ test providing a probability $\mathrm{p}$ for $\mathrm{z}$ being smaller than a critical value $\mathrm{z}_{\text {critical }}= \pm 1.96$. Differences with $\mathrm{p}$ values larger than 0.05 were considered statistically insignificant.

However, since the researchers interested in parchment analyses are mostly historians and thus are familiar with using calendar year ages, we also present the differences in calendar-year scale. We simulated the calendar year probability distributions (cpd) reflecting the years AD 1484 and 1506 with OxCal and the differences (Difference() command) between these 


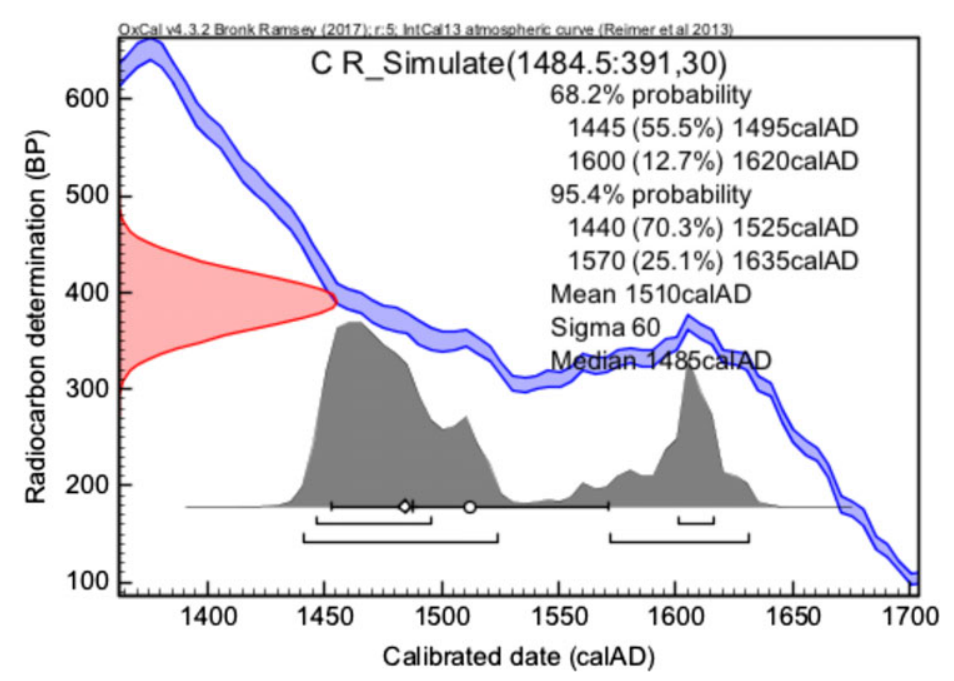

Figure 1 Example of a simulated ${ }^{14} \mathrm{C}$ date of the calendar year AD 1484 by assuming $\pm 30{ }^{14} \mathrm{C}$ year statistical uncertainty. Note that due to double-peak distribution, the mean value of the calibrated age is $1510 \pm 60$ calAD which is slightly later than the assumed age of AD 1484.

simulated cpds and the measured cpd were deduced to obtain the average of the 20 mean differences. This provided a way to estimate quantitatively the quality of the ${ }^{14} \mathrm{C}$ dating in calendar-year scale.

An example of a simulated ${ }^{14} \mathrm{C}$ date and its calibration is shown in Figure 1. Altogether, 220 simulations were made for AD 1484 and the average of the mean values of the obtained cpds was $1530 \pm 30 \mathrm{calAD}$. So, the average of means is slightly later compared to the assumed true age of $\mathrm{AD} 1484$. This seems to be due to variations in the atmospheric ${ }^{14} \mathrm{C}$ concentration causing a double-peaked shape of cpd. Nevertheless, the mean values of cpds provide a reasonable way to compare the quality.

\section{RESULTS AND DISCUSSION}

The first three series $(\mathrm{A}-\mathrm{C})$ were made during the progress of the method development on modern (A), 1506 (B) and 1484 (C) parchments (Table 1). Based on these, the chemical pretreatment process was refined and the remaining two series $(\mathrm{D}, \mathrm{E})$ were processed using the refined pretreatment process and with two combustion/graphitization processes.

\section{A-C Series}

The first series were characterized by development for chemical pretreatment and for the EA-HASE-AMS process itself. Therefore, we discuss the results of these series only briefly.

The measured pMC values for the A series ranged from 100.8 to 104.1. The majority of the deviations from the expected value for 2015 (101.6) can probably be connected to technical issues with the AMS target holder and cathodes. However, interestingly, both the largest $(2.4 \mathrm{mg} \mathrm{C})$ and the smallest $(0.12 \mathrm{mg} \mathrm{C})$ sample provided results that are closest to the assumed value of $101.2 \pm 0.3$ and $100.8 \pm 0.6 \mathrm{pMC}$. One of the most probable 
Table 1 Sample data for ${ }^{14} \mathrm{C}$-AMS measurements of parchments, $\mathrm{m}_{\text {sample }}=$ original mass of the parchment sample, $\mathrm{m}_{\mathrm{comb}}=$ parchment mass for combustion process, method = combustion \& graphitization method (CTC/EA-HASE), $\mathrm{m}_{\mathrm{C}}=$ estimated mass of graphite target for ${ }^{14} \mathrm{C}$-AMS measurements by assuming carbon content of $35 \%$ for collagen.

\begin{tabular}{llccclc}
\hline Sample ID & Material & Size $\left(\mathrm{mm}^{2}\right)$ & $\mathrm{M}_{\text {sample }}(\mathrm{mg})$ & $\mathrm{m}_{\text {comb }}(\mathrm{mg})$ & Method & $\mathrm{m}_{\mathrm{C}}(\mathrm{mg})$ \\
\hline A series & AD 2015 & & & & EA-HASE & \\
B series & AD 1506 & & & & EA-HASE & \\
C series & AD 1484 & & & & EA-HASE & \\
D1 & AD 1484 & 15 & 3.5 & 3.4 & CTC & 1.2 \\
D2 & AD 1484 & 12 & 2.7 & 2.6 & CTC & 0.9 \\
D3 & AD 1484 & 8.75 & 2.2 & 2.1 & CTC & 0.7 \\
D4 & AD 1484 & 6 & 1.7 & 1.5 & CTC & 0.5 \\
D5 & AD 1484 & 3 & 1.1 & 0.9 & CTC & 0.3 \\
D6 & AD 1484 & 27.5 & 7.0 & 6.7 & CTC & 2.3 \\
E1 & AD 1484 & 15 & 4.0 & 3.7 & EA-HASE & 1.3 \\
E2 & AD 1484 & 14 & 3.0 & 2.8 & EA-HASE & 1.0 \\
E3 & AD 1484 & 7.5 & 2.1 & 2.0 & EA-HASE & 0.7 \\
E4 & AD 1484 & 3 & 2.1 & 1.8 & EA-HASE & 0.6 \\
E5 & AD 1484 & 3 & 1.0 & 0.8 & EA-HASE & 0.3 \\
E6 & AD 1484 & 27.5 & 7.0 & 6.7 & EA-HASE & 2.3 \\
\hline
\end{tabular}

contaminants within parchment ${ }^{14} \mathrm{C}$ analyses can be carbonates as a lime bath was part of the process for the skins for hair removal. This contamination should be seen as decreased ${ }^{14} \mathrm{C}$ content in the data. There is no such indication within the data of modern parchment.

The series B made from AD 1506 parchment resulted in partially acceptable results as the two largest samples showed reasonably good agreement with the assumed age. However, the 4 measurements of the smaller samples $(0.77-0.15 \mathrm{mg} \mathrm{C})$ were all too old (mean values of cpds) by ca. 100-440 calendar years. Similarly, the C series made of AD 1484 parchment showed too old results as 3 out of 6 clearly had a ${ }^{14} \mathrm{C}$ concentration that was too small.

Based on the majority of the results (7/12) being too old, we tentatively linked this effect to carbonate (limestone) contamination, as this may have varied depending on the sampling location. Consequently, the chemical pretreatment was tuned to be longer in acid treatment to attack the potential limestone contamination. In addition, the $\mathrm{NaOH}$ treatment was made longer as it did not risk the sample survivals. We note that acetone wash can also be considered as a source of old carbon and, if present, the removal would require additional methanol/ethanol washes.

\section{${ }^{14} \mathrm{C}$ Concentrations in $\mathrm{D}$ and $\mathrm{E}$ Series}

For the final series D and E, the adjusted chemical pretreatment method was used on the 1484 parchment. To have a comparison to the well-established combustion/graphitization process, the D series were produced with the CTC process, whereas the E series was using the expectedly better-performing EA-HASE. 


\section{$114 T$ M Kasso et al.}

Table 2 Measurement data of the $\mathrm{D}$ and $\mathrm{E}$ parchment series. $\mathrm{RA}=$ radiocarbon age. $\sigma_{\mathrm{RA}}=$ uncertainty of the RA $(1 \sigma)$. Difference $=$ average of the mean differences of 20 simulated cpd and the measured one. $\sigma_{\text {Diff }}=$ standard deviation of Difference.

\begin{tabular}{lcccc}
\hline Sample ID & RA $(\mathrm{BP})$ & $\sigma_{\text {RA }}$ & Difference & $\sigma_{\text {Diff }}$ \\
\hline D1 & 406 & 33 & 31 & 23 \\
D2 & 374 & 35 & -4 & 32 \\
D3 & 356 & 34 & -7 & 25 \\
D4 & 391 & 32 & 13 & 24 \\
D5 & 30 & 108 & 17 \\
D6 & 387 & NA & -7 & 38 \\
E1 & 375 & 36 & $\mathrm{NA}$ & $\mathrm{NA}$ \\
E2 & $\mathrm{NA}$ & 36 & -38 & 35 \\
E3 & 323 & 33 & 34 & 36 \\
E4 & 421 & 31 & 111 & 20 \\
E5 & 488 & 34 & 23 & 30 \\
E6 & 385 & & 6 & 29 \\
\hline
\end{tabular}

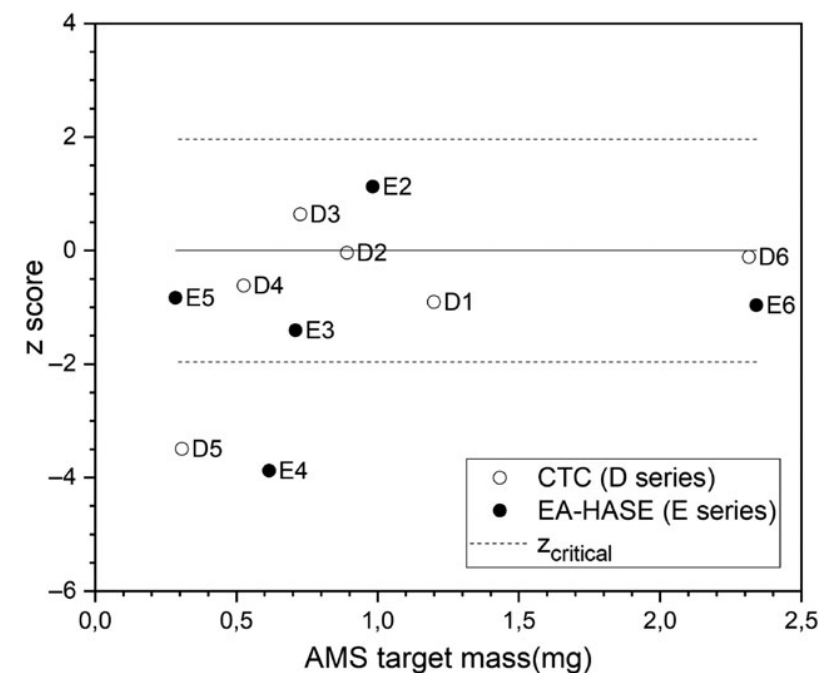

Figure 2 Comparison of measured and simulated pMC values for $\mathrm{D}$ and $\mathrm{E}$ series. The $\mathrm{z}$ score analysis shows two statistically significant outliers (D5 and E4). Differences between the measured and simulated results are not statistically significant for most of the samples.

The measurement data on the D and E series are given in Table 2. Overall, the z-score analysis (Figure 2) shows that for 9 out of 11 samples there is no statistically significant difference between the measured and simulated pMC values, as the $\mathrm{z}$ scores remain within the critical values. These series demonstrate that prolonging the chemical pretreatment process works as expected: the true age is reproduced better compared to series $\mathrm{B}$ and $\mathrm{C}$ indicating that there is, indeed, an issue of carbonate contamination within parchments. 


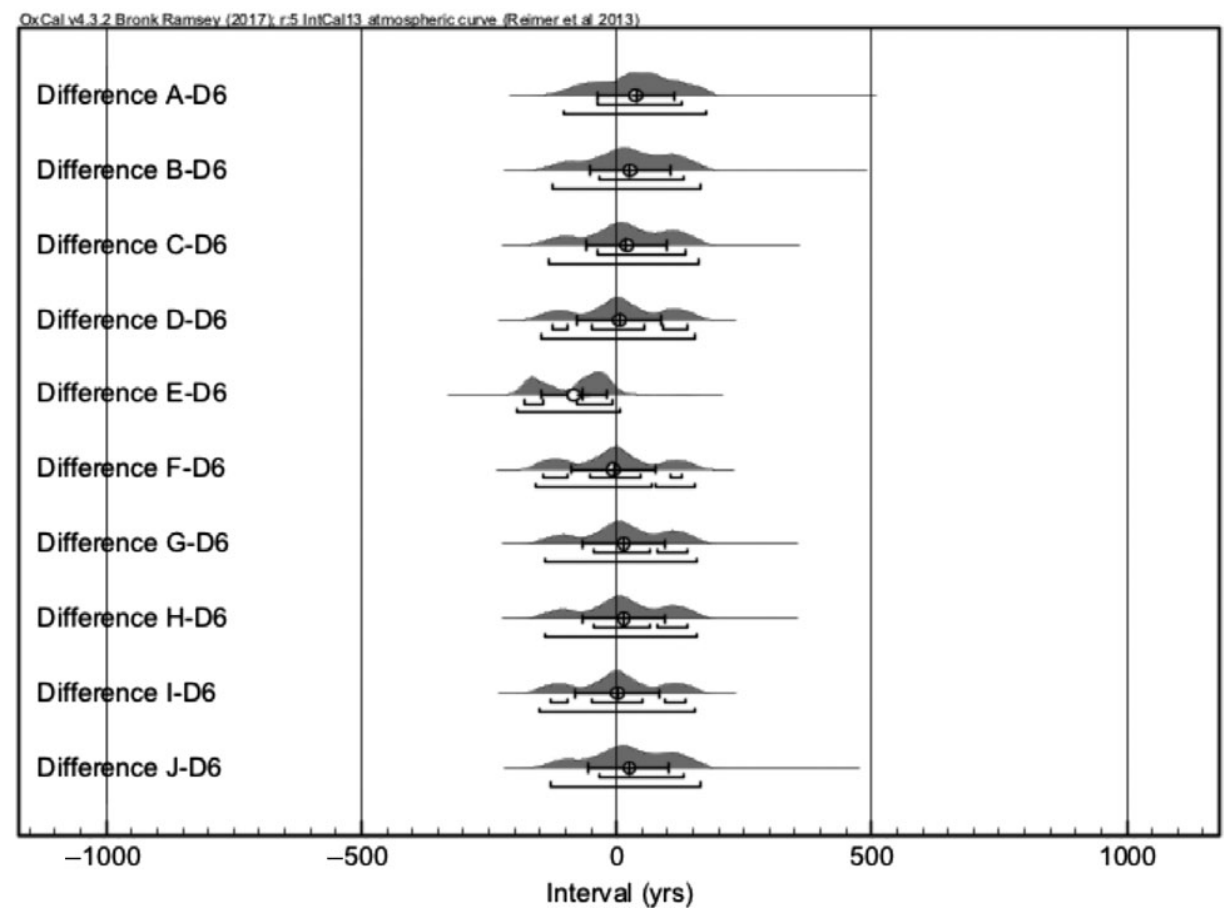

Figure 3 An example of a comparison of calendar year distributions of 10 simulated ${ }^{14} \mathrm{C}$ ages of $\mathrm{AD}$ 1484 and the measurement of D6 sample.

However, the analysis also pinpoints two statistically significant outliers in the data: D5 and E4 samples. For these, the measured pMC values are too low by $3-4$ standard deviations. It is well-known that small samples may be influenced by residual- $\mathrm{CO}_{2}$ contamination from the vacuum lines and cause ages to become too young due to modern $\mathrm{CO}_{2}$ involvement (Donahue et al. 1990). We do not see such a modern contamination effect, indicating that the potential contamination of residual gas- $\mathrm{CO}_{2}$ for medieval samples is well in control for both processes. On the contrary, the only deviating concentrations (D5 and E4) are too low. As the clean room excludes external contamination from pretreatment, since our process and AMS backgrounds are reasonably low, and since prolonging the chemical pretreatment seems to work as expected, we tentatively connect this effect to remaining carbonate contamination in these samples.

\section{Results in a Calendar Year Scale}

Figure 3 shows the comparison of the result of the largest sample (D6 with $2.3 \mathrm{mg}$ graphite mass) to 10 simulated cpds of the year 1484. The figure shows also why one needs to make a large enough number of simulations and averaging of simulated cpds as the one of the simulations (simulation E) produces clearly a deviant cpd.

The simulated cpds and the ${ }^{14} \mathrm{C}$ date of D6 sample (Figure 4) generally show a reasonable agreement as the Difference distributions are mostly centered around 0 . This converts to (Table 2) an average difference between the simulations and measurement as $-7 \pm 38$ calendar years (cal yr). Similarly, the samples D1-D4 provide also average difference 


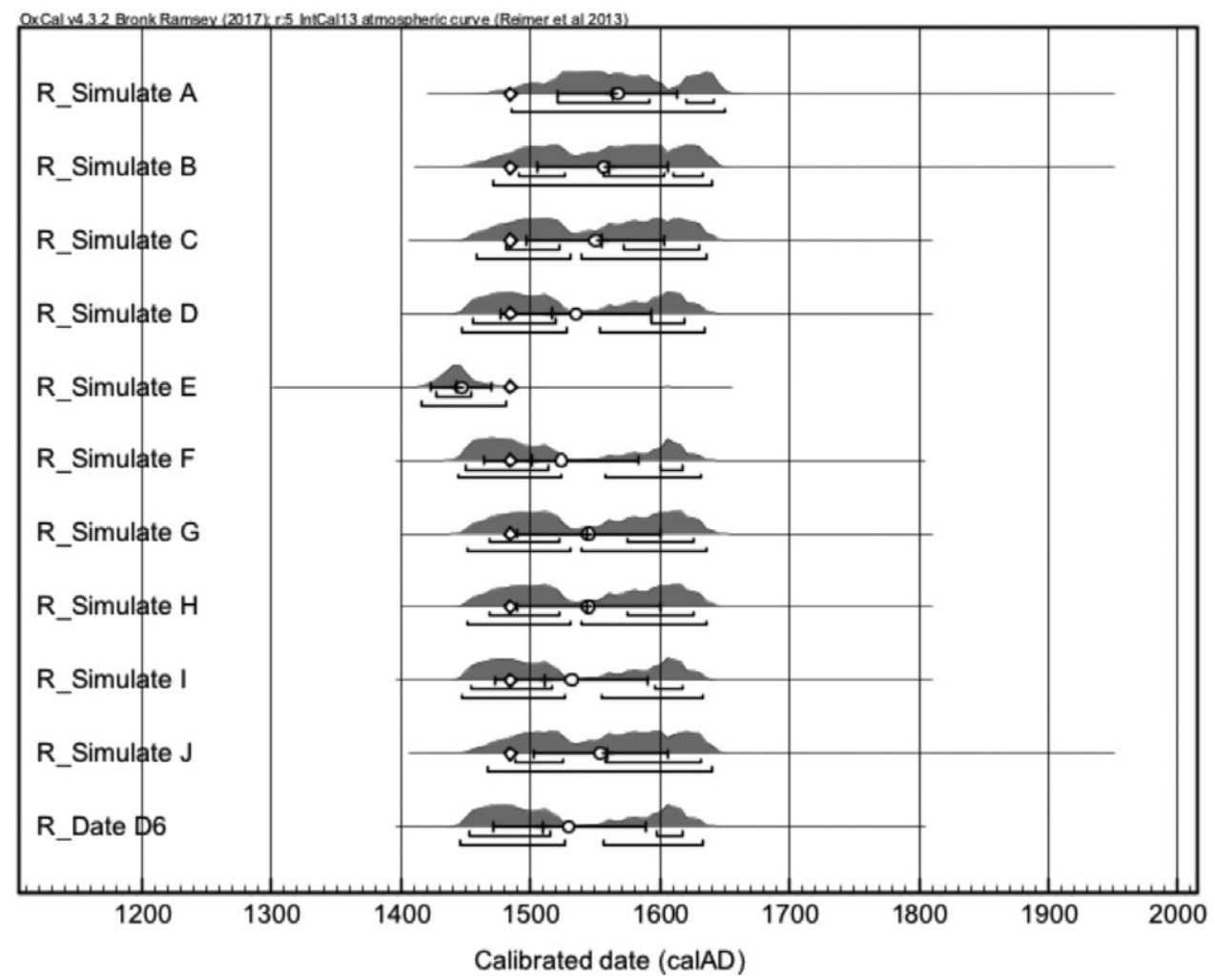

Figure 4 Differences between 10 simulated calendar year probability distributions of AD 1484 and the measurement D6 in calendar years. Eventually, 20 simulations and corresponding differences were made for each comparison.

distributions centered around 0 . Thus, we conclude that the ${ }^{14} \mathrm{C}$ dates of the parchment samples D1-4, 6 reproduce the true age of the sample well. Overall, the CTC-AMS process can reproduce the known ages for at least as low as $\sim 0.5 \mathrm{mg}$ AMS target sizes.

The E series demonstrated a similar trend according to sample size for the EA-HASE-AMS process. Similar to the D series, simulated cpds for the year 1484 show a slight scattering (Figure 5) that emphasize the importance of the averaging of simulated distributions. The measurement of the largest sample E6 produces a two-peak distribution of which the largest probability mass falls at the end of the 15th century, as expected.

The differences between the simulated cpds and the ${ }^{14} \mathrm{C}$ date of E6 sample (Figure 6) show a similar, better agreement than D6 as the Difference distributions are mostly centered around 0. Again, this converts to an average difference of $6 \pm 29$ cal yr between the simulated and the measured cpds. The E1 sample was lost due to technical issues, but the E2,3 and 5 samples show similar good agreement with the simulations. The only exception is the sample E4 $\left(\mathrm{m}_{\mathrm{C}}=0.6 \mathrm{mg}\right)$ for which the low ${ }^{14} \mathrm{C}$ concentration converts to a date ca. $100 \mathrm{cal} \mathrm{yr}$ too old. Particularly, the measurement of the smallest sample E5 with $0.3 \mathrm{mg}$ sample size reproduced well the true age of the parchment. This target mass corresponds typically to parchment samples of ca. $3 \mathrm{~mm}^{2}$ size in area (Table 1). 


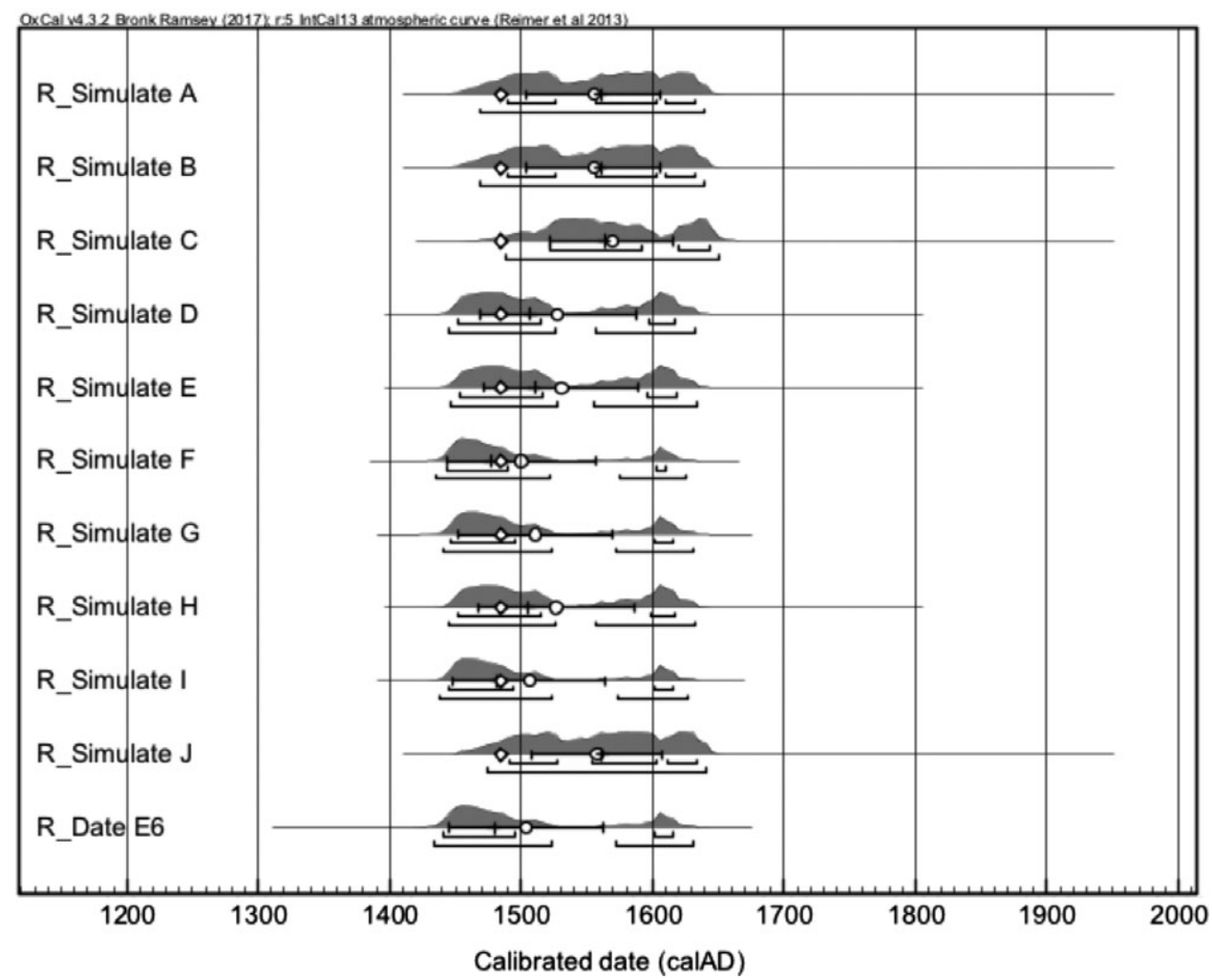

Figure 5 An example of a comparison of calendar year distributions of 10 simulated ${ }^{14} \mathrm{C}$ ages of AD 1484 and the measurement of E6 sample.

Overall, the simulated age of the 1484 parchment is reproduced (Figure 7) throughout the sample sizes. This shows that the ${ }^{14} \mathrm{C}$ dating results of series D-E were satisfactory. Whereas the difference between the CTC and EA-HASE is tenuous, the less laborious EA-HASE-AMS process matches the expectations by providing excellent result with slightly smaller AMS target mass of $0.3 \mathrm{mg}$, corresponding to ca. $3 \mathrm{~mm}^{2}$ parchment areas. Moreover, it provides lower radiocarbon background also crucial for small sample measurements. In the future, sample purity with a focus on the carbonate contamination in unknown samples will be addressed by applying Fourier transform infra-red (FTIR) spectroscopy. Additionally, following the results presented in this paper, we aim to improve chronological analyses of the Fragmenta membranea samples with exploring even smaller $>0.3 \mathrm{mg} \mathrm{C}$ samples sizes for parchment.

\section{CONCLUSION}

The aim of this study was to determine the lowest sample size for ${ }^{14} \mathrm{C}$ dating of parchment, and in particular to resolve whether it is possible to decrease the typical amount of carbon ( $1 \mathrm{mg})$ needed for ${ }^{14} \mathrm{C}$ dating. Focus was placed on moving the chemical pretreatment process to a clean room to exclude surface contamination during the pretreatment. The process was developed successfully through three test series with modern and medieval parchments of known ages. Eventually, both of the processes tested-the traditional Closed-Tube-Combustion 


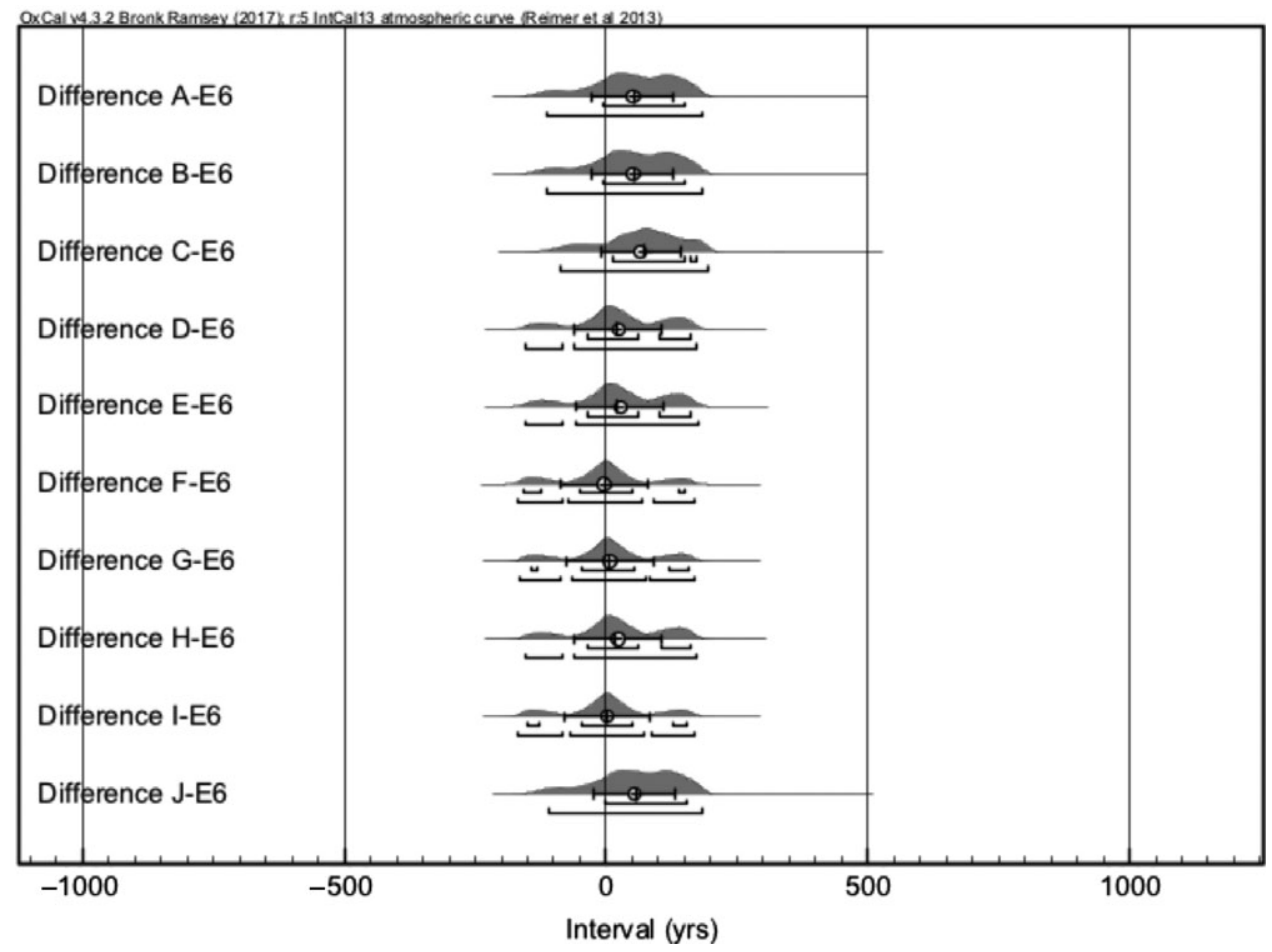

Figure 6 Differences between simulated calendar year probability distributions of AD 1484 and the measurement E6 in calendar years. Eventually, 20 simulations and corresponding differences were made.

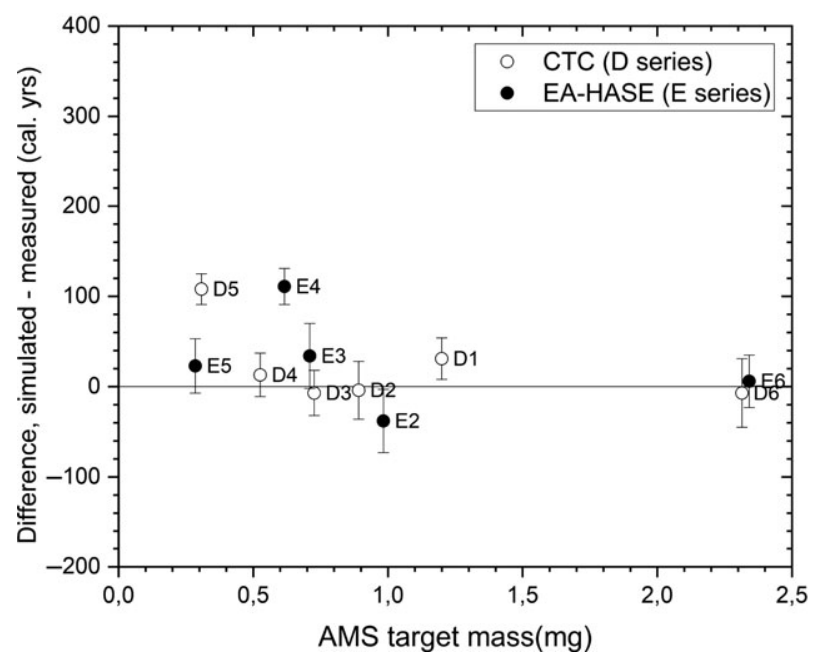

Figure 7 Differences between all the simulated and measured calendar year dates as a function of the AMS target masses. 
\& graphitization (CTC) and Elemental Analyzer combustion and automated graphitization (EA-HASE) - were demonstrated to work with significantly smaller sample sizes. Particularly, EA-HASE-AMS process was so far able to reproduce the true age of the sample down to $0.3 \mathrm{mg} \mathrm{C}$ AMS target mass corresponding to parchment samples of ca. $3 \mathrm{~mm}^{2}$ and $0.8-1 \mathrm{mg}$ before pretreatment. Therefore, this destructive method of analysis can be applied within a range to limited material, or material which does not allow large sample amounts. The information obtained pertaining to the necessary sample size can also be used in outlining the research of historical material that has perhaps been left untouched, because its sampling has been previously considered too invasive.

In the future, we will proceed towards exploiting the full potential of the EA-HASE-AMS process with $50 \mu \mathrm{g}$ AMS graphite target masses and expand these initial studies to sample quality criteria with FTIR method in order to better understand the role of contaminants, such as carbonates within the parchment samples due to the manufacturing process with lime. In addition to this, we will extend our studies to the Fragmenta membranea parchment fragment collection. Furthermore, we will combine the datable information from historical and paleographic studies with Bayesian modelling to produce a multidisciplinary chronological analysis of parchment archives. Dating and studying history should be viewed as a multistage process, in which the one method is not ignored for another, but applied together.

\section{ACKNOWLEDGMENTS}

The study is part of the Towards Reliable Dating of Medieval Parchments: Developing Traditional and Scientific Methods project, funded by The Jenny and Antti Wihuri Foundation, and collaboration between the Laboratory of Chronology of the Finnish Museum of Natural History (LUOMUS) and the National Library of Finland. Additionally, the first author was a member of the Laboratory of Chronology in Helsinki when this research was undertaken.

\section{REFERENCES}

ASTM. 2018. Standard test methods for determining the biobased content of solid, liquid, and gaseous samples using radiocarbon analysis. ASTM D 6866-18. West Conshohocken (PA): ASTM International.

Bonani G, Ivy S, Wölfli W, Broshi M, Carmi I, Strugnell J. 1992. Radiocarbon dating of fourteen Dead Sea Scrolls. Radiocarbon 34(3): 843-849.

Brock F, Dee M, Hughes A, Snoeck C, Staff R, Bronk Ramsey C. 2018. Testing the effectiveness of protocols for removal of common conservation treatments for radiocarbon dating. Radiocarbon 60(1):35-50.

Brock F. 2013a. Radiocarbon dating of historical parchments. Radiocarbon 55(2-3):353-363.

Brock F. 2013b. Preparing samples for AMS radiocarbon dating. In: Shortland AJ, Bronk Ramsey C, editors. Radiocarbon and the chronologies of Ancient Egypt. Oxford: Oxbow Books. p. 48-52.

Brock F, Higham T, Ditchfield P, Bronk Ramsey C. 2010. Current pretreatment methods for AMS radiocarbon dating at the Oxford Radiocarbon

Accelerator Unit (ORAU). Radiocarbon 52(1):103-112.

Brock F, Wood R, Higham T, Ditchfield P, Bayliss A, Bronk Ramsey C. 2012. Reliability of nitrogen content $(\% \mathrm{~N})$ and carbon: nitrogen atomic ratios $(\mathrm{C}: \mathrm{N})$ as indicators of collagen preservation suitable for radiocarbon dating. Radiocarbon 54(3-4):879-886.

Bronk Ramsey C. 2009. Bayesian analysis of radiocarbon dates. Radiocarbon 51(1): 337-360.

Donahue DJ, Linick TW, Jull AJT. 1990. Isotoperatio and background corrections for accelerator mass spectrometry radiocarbon measurements. Radiocarbon 32(2):135-142.

Donahue DJ, Olin JS, Harbottle G. 2002. Determination of the radiocarbon age of parchment of the Vinland Map. Radiocarbon 44(1):45-52.

Gupta SK, Polach HA. 1985. Radiocarbon dating practices at ANU. Canberra: Australian National University Research School of Pacific Studies.

Hedges REM, van Klinken GJ. 1992. A review of current approaches in the pretreatment of bone 
for radiocarbon dating by AMS. Radiocarbon 34(3):279-291.

van Klinken GJ. 1999. Bone collagen quality indicators for palaeodietary and radiocarbon measurements. Journal of Archaeological Science 26(6):687-695.

Levin I, Kromer B, Hammer S. 2013. Atmospheric $\Delta^{14} \mathrm{CO}_{2}$ trend in Western European background air from 2000 to 2012. Tellus B: Chemical and Physical Meteorology 65:1, 20092.

Palonen V, Pesonen A, Herranen T, Tikkanen P, Oinonen M. 2014. HASE - The Helsinki Adaptive Sample Preparation Line. Nuclear Instruments and Methods in Physics Research. Section B: Beam Interactions with Materials and Atoms 294:182-184.

Rasmussen KL, van der Plicht J, Doudna G, Nielsen F, Højrup P, Stenby EH, Pedersen CT. 2009. The effects of possible contamination on the radiocarbon dating of the Dead Sea Scrolls
II: empirical methods to remove castor oil and suggestions for redating. Radiocarbon 51(3): 1005-1022.

Slota P, Jull A, Linick T, Toolin L. 1986. Preparation of small samples for ${ }^{14} \mathrm{C}$ accelerator targets by catalytic reduction of CO. Radiocarbon 29(2): 303-306.

Santos FJ, Gómez-Martínez I, García-León M. 2010. Radiocarbon dating of medieval manuscripts from the University of Seville.

Scott E, Cook G, Naysmith P. 2010. The fifth international radiocarbon intercomparison (VIRI): an assessment of laboratory performance in stage 3. Radiocarbon 53(2-3):859-865.

Tikkanen P, Palonen V, Jungner H, Keinonen J. 2004. AMS facility at the University of Helsinki. Nuclear Instruments and Methods in Physics Research B 223-224:35-39.

Uusitalo J, Mizohata K, Oinonen M. 2020. In preparation. 